\title{
Effect of Simultaneous Emotions and Driving Tasks on Driver's Change Blindness
}

\author{
Yanqun Yang1, Xinyi Zheng2*, Said Easa ${ }^{3}$, Qian Zhang², Aixiu Hu1, Yu Liang1 \\ ${ }^{1}$ School of Civil Engineering, Fuzhou University, Fuzhou, China \\ ${ }^{2}$ Faculty of Humanities and Social Sciences, Fuzhou University, Fuzhou, China \\ ${ }^{3}$ Faculty of Engineering and Architectural Science, Ryerson University, Toronto, Canada \\ Email: ${ }^{*}$ zhengxinyi@fzu.edu.cn
}

Received 26 May 2014; revised 5 July 2014; accepted 15 August 2014

Copyright (C) 2014 by authors and OALib.

This work is licensed under the Creative Commons Attribution International License (CC BY). http://creativecommons.org/licenses/by/4.0/

(c) (i) Open Access

\begin{abstract}
Change blindness is an important phenomenon where a driver may skip some information in the driving scene due to its rapidly changing and dynamic character. The purpose of this paper is to examine the effect of simultaneous emotions and driving tasks on change blindness. Twenty college students with driving licenses were taken as the study subjects. The experimental program involved two types of emotions combined with four driving tasks. Showing 10 minutes movie to arouse two types of emotions was one methodology and using E-prime software to show one-shot paradigm of change blindness was the other. The results show that emotion and driving tasks affect change blindness. The subjects took longer time to perceive the changes in the mood of negative emotion than they did in the mood of positive emotion. The level of change detection was found to decrease after the driving tasks were performed. Furthermore, the tasks with traffic signs showed more change blindness than those without traffic signs. The groups performing the turning-right tasks exhibited more change blindness than those performing the going-ahead tasks. The practical contributions of the present study are discussed in relation to the human-oriented design of traffic signs.
\end{abstract}

\section{Keywords}

Driving Tasks, Emotions, Road Scenes, Experiments, Change Blindness

Subject Areas: Psychology, Sociology

\section{Introduction}

The impact of change blindness on our lives is exceedingly strange, and usually people are hard to realize this

*Corresponding author.

How to cite this paper: Yang, Y.Q., Zheng, X.Y., Easa, S., Zhang, Q., Hu, A.X. and Liang, Y. (2014) Effect of Simultaneous Emotions and Driving Tasks on Driver's Change Blindness. Open Access Library Journal, 1: e868.

http://dx.doi.org/10.4236/oalib.1100868 
effect. Traffic collision studies indicate that the error distribution of attention is the main cause of collisions [1] [2]. Most collisions happened because of a too-late notice or no notice at all of critical information in the road scene [3].

Theoretical values of change blindness have attracted much attention for several decades such that it becomes a research hotspot. Research on change blindness in the literature has ranged from the study of scattered verified phenomena to the study of information integration of the real scene [4] [5]. Previous studies suggested that change blindness referred to the observer's awareness of the changes between two scenes, but are imperceptible changes of an object or scene due to the flicker, blink of an eye, scanning, or film switching [6]. Even if the eyes are fixed on the vicinity of the position where change occurs, the observer cannot perceive this change. At present, change blindness is defined as a situation in which the driver is not effectively able to detect changes in the rapidly changing and dynamic environment [7]. Change blindness occurs because the observer, first of all, focuses his/her attention on the meaning of the visual scene when looking for a change. The focus of attention is directed by the target and enables the observer to use cognitive resources (strategies) to quickly find related information within the limited space [6].

Several researchers have studied the effect of driving tasks on change blindness. Koustanai, Elslande \& Bastien (2012) [8] explored the main effects and the interaction of left-turn driving in France (equivalent to right-turn driving in China and North America), environmental landscape, and level of participant driving experience in the experiment. Change blindness affects driver's use of cognitive strategy in actual situations. Previous studies show that even very large changes may not be noticed if they are not the focus of attention. Besides, the mood of emotion was found to affect the scope of attention. Studies on emotions have shown that positive emotions broaden the scope of attention and thought-action repertoires, while negative emotions narrow these repertoires [9]. Because positive emotion produces "broad, flexible cognitive organisation and ability to integrate diverse material" [10], the effects are linked to increases in brain dopamine levels, particularly in the prefrontal cortex and anterior cingulate, which are thought to underlie better cognitive performance [11]. As such, it was believed that positive emotions promote task-switching attention as a result of detecting environmental changes more efficiency than negative emotions do.

Recent research has examined the effect of simultaneous emotions and normal cognitive tasks [12]. The results showed that positive emotions play a promoting role in the cognitive task and negative emotions hinder the individual to perceive changes. Moreover, the influence of emotion on change detection was mediated by cognitive task difficulty, where positive emotion aids change detection in difficult tasks, while emotion states have no effect on change detection in easy tasks. In recent years, studies on change blindness have made substantial development [13], but the research on the effect of simultaneous emotions and driving tasks is lacking. Traditional cognitive processing research usually excludes emotional factors to more accurately reproduce the human information processing stage. In fact, emotions and cognition interaction complement each other.

The present study aimed at determining the effect of simultaneous emotion and driving tasks on change blindness. The experimental program involved four driving tasks combined with two states of emotions. The driving tasks were: going ahead (AHEAD-task), turning right (RIGHT-task), going ahead under guiding signs (AHEADStask), and turning right under guiding signs (RIGHTS-task). The two states of emotions were: positive or happy emotion (P-emotion) and negative or angry emotion (N-emotion). The hypotheses of the study are as follows: 1 ) different driving tasks may affect different abilities of change blindness (time and accuracy of change detection), 2) the state of emotional arousal affects the level of change blindness, and 3) the level of change blindness is different in the two states of emotions of experimental measurement, where drivers with positive emotions more easily detect changes in the driving scene and those with negative emotions are prone to change blindness.

The following section presents the research methodology, including participants, experimental materials, and experimental process. The research results and discussion are then presented, followed by conclusions.

\section{Research Methodology}

\subsection{Participants}

Twenty eight students from Fuzhou University (aged 20 to 24) whose driving experience was less than two years were randomly divided into two groups. One group was aroused with P-emotion and the other was aroused with $\mathrm{N}$-emotion. After the arousal phase, eight students were discarded from the experiments since their arousal was not adequate, as discussed later. The final experiment included 10 students (5 males and 5 females) in each 
group.

\subsection{Experimental Materials}

The experimental materials included participant questionnaire, initial change blindness materials, emotional arousal materials, and final experiment materials. The participant questionnaire is mainly used for collecting the driving years of the participants in order to filter them. A description of the other three types of materials is presented next.

\subsubsection{Initial Change Blindness Materials}

Materials for assessing the initial change blindness included 40 photos with different transport-related scenarios, 20 of them were selected to manipulate with photoshop software by inserting or deleting the traffic-related scenarios, such as a car, pedestrians, traffic lights. The Eprime software was used in the experiment.

\subsubsection{Emotional Arousal Materials}

These materials included videos that can effectively lead to P-emotion and N-emotion. For N-emotion, several local studies have confirmed that The Tokyo Trial is the appropriate film material to induce anger emotion (this film presents scenes related to the Japanese invasion of China in World War II). For P-emotion, two video clips that could trigger the required emotions were preliminary selected from the literature: Chrysanthemum to the Beast and Lost on Journey, The screening process to determine the best video was as follows. After watching the videos, the participant was asked to fill in an emotional evaluation form that included six kinds of emotional intensities. For each kind, the participant recorded a score from 0 to 6 (Likert 7 scale), where 0 means no emotional intensity and 6 means high emotional intensity. The scores were then statistically analyzed.

The effects of the video induced emotion were evaluated using three measures: induced intensity, hit rate, and statistical significance (Table 1). The induced intensity refers to the average induced emotions, including target and non-target emotions. The higher the value of the target emotion is, the higher the intensities of the induced emotion. As noted, the induced intensity of the happiness emotion is higher than that of other emotions in the two films and the induced intensity of the happiness emotion in Lost on Journey is better than that in Chrysanthemum to the Beast $(4.80>4.47)$. The other two statistical measures also supported the selection of Lost on Journey. Therefore, the two videos selected for conducting the final experiment were Lost on Journey and The Tokyo Trial (each video was watched by the participants for about 20 minutes).

\subsubsection{Final Experiment Materials}

The final experiment design involves 80 pairs of photos with four different driving tasks. Each task consisted of 20 pairs of photos.

\subsection{Experimental Process}

The experiment was divided into five phases: 1) Filtering participants, 2) assessing initial change blindness, 3) creating emotional arousal, 4) conducting final experiment, and 5) processing data. In Phases 2 and 4, a one-shot paradigm, in which one each scene included only a single change, was applied in the experiments.

Table 1. Comparison of induced positive emotion intensity and uniqueness for two videos.

\begin{tabular}{|c|c|c|c|c|c|c|c|}
\hline \multirow{2}{*}{ Video } & Happiness & Anger & Sadness & Fear & Surprise & Disgust & Hit Rate \\
\hline & \multicolumn{7}{|c|}{ a) Score of Emotion Intensity } \\
\hline Chrysanthemum to the Beast & 4.47 & 0.73 & 0.07 & 0.67 & 1.33 & 1.20 & 0.8 \\
\hline \multirow[t]{2}{*}{ Lost on Journey } & 4.80 & 0.20 & 0.07 & 0.07 & 1.60 & 0.47 & 0.93 \\
\hline & \multicolumn{7}{|c|}{ b) Score of Emotion Uniqueness } \\
\hline Chrysanthemum to the Beast & $--^{a}$ & 0.36 & 0.87 & 0.09 & 0.89 & 0.63 & $--^{\mathrm{a}}$ \\
\hline Lost on Journey & $--^{\mathrm{a}}$ & 0.00 & 0.00 & 0.00 & 0.54 & 0.49 & $-{ }^{\mathrm{a}}$ \\
\hline
\end{tabular}

${ }^{\mathrm{a}}$ Not applicable. 
1) Filtering Participants: Twenty eight participants with two-year driving experience were selected from Fuzhou University campus. The participants were randomly divided into two groups, one group for P-emotion and the other for N-emotion. Eight participants were eliminated because they did not achieve the required level of emotional arousal (Phase 3).

2) Assessing Initial Change Blindness: This phase involved the following procedures: a) present the masking stimulus “+” for $3 \mathrm{~s}, \mathrm{~b}$ ) randomly select and present the original photo for $3 \mathrm{~s}, \mathrm{c}$ ) present a blank screen for $1 \mathrm{~s}$, and d) present the changed photo or the original photo for $3 \mathrm{~s}$. The change in the target photo is related to traffic operation (e.g. cars, passengers, or traffic lights). The participant was asked to press a key to judge whether the two photos presented before and after were the same or different. The participant then pressed the $\mathrm{F}$ or $\mathrm{J}$ key, if he/she perceived or did not perceive a change, respectively. The Eprime software recorded the reaction time (R) and accuracy in percentage (A) for each participant. These measures are defined as follows:

$$
\begin{gathered}
R=T_{2}-T_{1} \\
A=100(n / N)
\end{gathered}
$$

where $T_{1}=$ time when the second photo goes off, time when the participant presses the $F$ or $J$ key, $T_{2}=$ time when the participant presses the $F$ or $J$ key, $n=$ number of photo pairs answered correctly, and $N=$ total number of photo pairs shown to the participant.

3) Creating Emotional Arousal: Before the final experiment, P-emotion group watched a segment (about 20 minutes) from the movie Lost on Journey and N-emotion group watched a segment from the movie The Tokyo Trial. Then, the participants reported whether they were in emotional arousal on a scale of 1 to 10 , where 1 means low arousal and 10 means high arousal. The participants who recorded emotional arousal of 5 or more were allowed to continue for the final experiment.

4) Conducting Final Experiment (Figure 1): The final experiment was conducted using the Eprime software. The paradigm adopted in the experiment was similar to that of Koustanai et al. (2012). The participant attention was dispersed by assigning the task before the start of the experiment. In this way, the participants would focus their attention on the completion of the task rather than on the emergence of a change, so they caused change blindness. As previously mentioned, the study included four task conditions combined with two types of emotions. In each experiment, the instructions were presented to the participants and they were asked to carefully read them. Then, the masking stimulate "+" is presented, followed by the original photos for $3 \mathrm{~s}$ (no task interference, and the photos of four kinds of tasks were presented randomly). Next, a blank photo is shown for $1 \mathrm{~s}$, followed by the original or the changed photo for $3 \mathrm{~s}$.

After each experiment, the participant was asked to evaluate the difficulty of completing the task in the lastpresented scene. The response options were: “easier” (no obstacles were encountered in achieving the task),

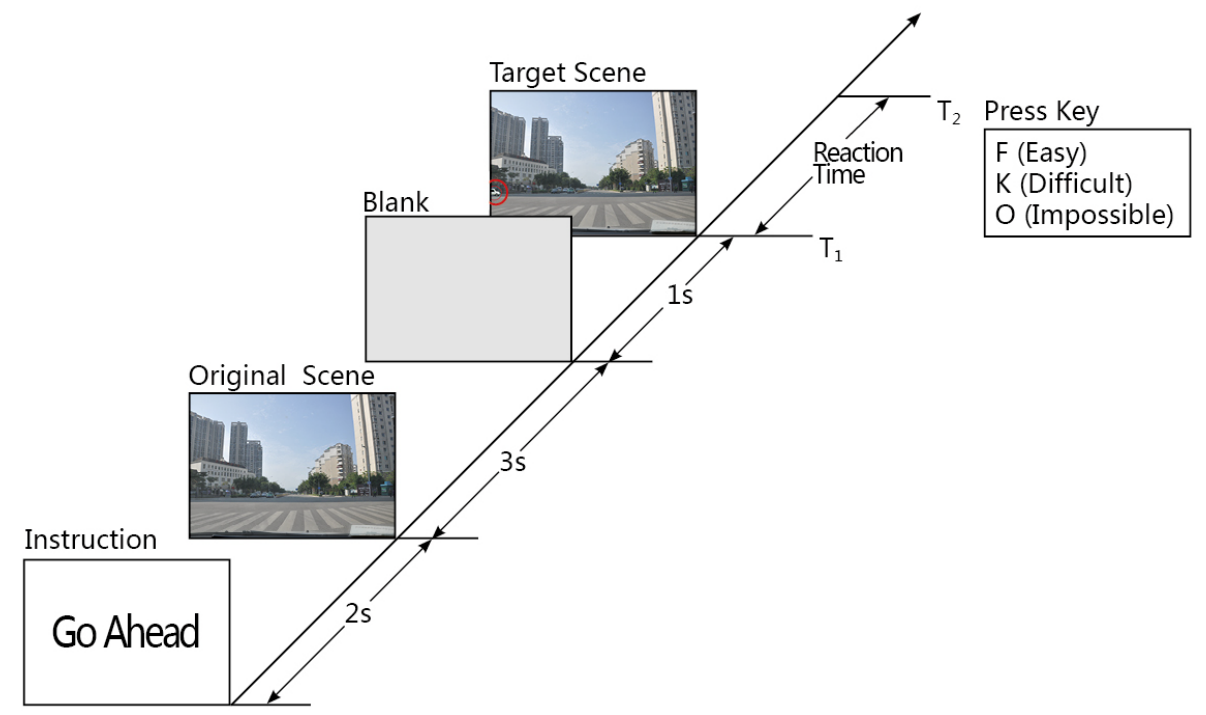

Figure 1. Sequence of the procedures in the final experiment. 
"more difficult" (an obstacle was encountered and prevented the achievement of the task promptly), or "impossible" (task could not be achieved). The participant completed the response by pressing the F, K, or O key, respectively. Then, the reaction time and accuracy were recorded. The participants chose the "impossible" option when the facilities in the photos were insufficient to make them achieve their goals. For example, the participants were required to drive according to the traffic signs suggested while there were no traffic signs in the photo.

5) Data Processing: The reaction time and accuracy data recorded using the E-prime software were organized. Then, the statistical analysis of the organized data was performed using the statistics software SPSS16.0.

\subsection{Results}

The effects of emotion and driving tasks on driver change blindness were evaluated. The initial change blindness between the two emotion groups was analyzed using the independent t-test (Table 2). It was found that the two groups were homogeneous for both reaction time $(t=0.346, p>0.05)$ and accuracy $(t=-0.763, p>0.05)$, so they were qualified to take part in the next phase the experiment. In addition, the two-way repeated ANOVA was applied to test the effects of various factors and their interactions on change blindness.

\subsection{Effect of Emotions on Change Blindness}

The difference between the change detection times of the initial and final experiments ( $2.199 \mathrm{~s}$ and $2.379 \mathrm{~s}$, respectively) was not found to be significant for P-emotion group $(t=0.817, p=0.435)$. For N-emotion group, the difference between the change detection times (2.088 s vs. $2.595 \mathrm{~s}$ ) was significant $(t=2.333, p=0.045)$. On the other hand, both emotional groups showed a significant impact on decreasing change detection accuracy between initial and final experiments (62\% - 65\% and 46\% - 47\%, respectively).

To better understand how much decreasing effect was statistically changed, the independent t-test was applied to test the difference between change detection times and accuracies of the final and initial experiments. As noted, the difference was not statistically significant for both indicators. These results suggest that driving change blindness is impacted by emotions (positive or negative) and both emotions equally impact the decrease in change detection accuracy. It was noticed that $\mathrm{N}$-emotion group required more time to detect the change than did P-emotion group.

\subsection{Effect of Driving Tasks on Change Blindness}

As previously mentioned, two categories of driving tasks were used: sign-guided tasks and direction-guided tasks. The results show that, compared to the initial level of change blindness, the driving tasks impacted the performance of change detection for either the reaction time or the accuracy of responding. Specifically, signguided driving tasks had longer change detection time than no sign-guided tasks (2.609 s and $2.361 \mathrm{~s}$ ), Compared with the initial change detection time, sign-guided tasks induced significantly longer reaction times than no-sign-guided tasks $(t=3.248, p<0.01)$. There was no significant difference in the change detection accuracy for both sign and no-sign-guided tasks.

For direction-guided tasks, turning-right tasks had longer change detection time than going-ahead tasks did (2.571 s vs. 2.399 s). Compared to the initial change detection time and accuracy, turning-right tasks induced significantly longer reaction times than going-ahead tasks, where $(t=3.954, p<0.01)$ and $(t=3.339, p<0.01)$, respectively. The change detection accuracy in turning-right tasks decreased more than that of going-ahead tasks (41\% vs. $52 \%)$.

Table 2. Comparison of reaction times and accuracies of the P-emotion and N-emotion groups in the final experiment ${ }^{\mathrm{a}}$.

\begin{tabular}{|c|c|c|c|c|c|}
\hline \multirow{2}{*}{ Indicator } & \multicolumn{3}{|c|}{ Mean \pm Standard Deviation } & \multirow{2}{*}{$t$-value } & \multirow{2}{*}{$p$-value } \\
\hline & P-emotion Group (PG) Difference & N-emotion Group (NG) Difference & Difference (PG - NG) & & \\
\hline Reaction time (s) & $-0.176 \pm 0.680$ & $-0.507 \pm 0.688$ & $-0.331 \pm 0.306$ & -1.084 & 0.293 \\
\hline Accuracy (\%) & $17 \pm 14$ & $18 \pm 8$ & $1 \pm 5$ & 0.256 & 0.801 \\
\hline
\end{tabular}

${ }^{\mathrm{a}}$ Degrees of freedom $=18$. 
To better understand the effect of change detection in the four driving tasks, a post-hoc comparison was applied to examine their interaction effects. For change detection time, there was no difference between RIGHTtask and AHEADS-task. The results of all other driving tasks showed a significant difference. For change detection accuracy, no difference was found for the right-turn tasks with and without signs. The results of all other driving tasks compared in pair were also significantly different.

In general, the change detection time increased differently when the driving task changed. RIGHTS-task required the longest change detection time than the other three driving tasks, followed by AHEADS-task, RIGHTtask, and AHEAD-task. In fact, there was no difference between RIGHT-task and AHEAD-task. Furthermore, for change detection accuracy the right-turn tasks with and without signs showed no significant difference. AHEAD-task had the least effect on change detection accuracy, followed by AHEADS-task, RIGHTS-task, and RIGHT-task. That is, change detection accuracy was less influenced by AHEAD-task than by RIGHT-task.

The analysis of the main and interaction effects of the driving tasks and emotions was also examined. The main effects of the driving tasks for reaction time and accuracy of the participants' responses were significant, where $(F(3,799)=9.294, p<0.05)$ and $(F(3,799)=5.013, p<0.01)$, respectively. The effect of emotion on change blindness was not significant for reaction time or accuracy, nor was the interaction effect of emotion and driving tasks.

\section{Discussion}

Change blindness is associated with attention and visual perception. Therefore, its paradigms are increasingly used in vehicle driving research because they are measuring how drivers process environmental information in their visual surrounding. The findings of such research could have important implications on transportation design and operation. The present study used a one-shot paradigm to compare the determinants of change detection as a function of both emotions and driving tasks. The study results have highlighted several important aspects of change blindness.

First, the results suggested that the accuracy of change detection was about $62 \%-65 \%$ in the initial experiment, compared with $46 \%$ - $47 \%$ in the final experiment. The difference was statistically significant. Such a difference is likely to be understood by the characteristics of change blindness. The ability of change detection is one measure of change blindness. It can also be explained by the visual attention hypothesis [14]. Although the visual system appears to be hardwired to detect these transients, they are not always present when something changes. In addition, visual changes can be remarkably difficult to detect under different conditions [5].

Many studies have explored what change detection failure really means. Does it mean that viewers did not represent visual information or they did represent it but failed to be aware of it? Generally, research suggests that viewers do, in some cases, represent visual information but may not do so automatically or indiscriminately. Therefore, it becomes critical to understand how visual attention is guided by knowledge, context, and visual characteristics. Some studies found that about $50 \%$ naïve participants were unable to detect the changes when the stimulus was displayed in static images [5] [15] and about 30\% of the observers failed to detect the changes when the stimuli was completely novel in shape and color [16]. Overall, the phenomenon of change blindness suggests that observers may not be completely aware of the attended stimulus and the amount of detected stimulus may vary under different conditions (e.g. naturalistic and experimental).

Second, the effect of emotion was found in the initial change blindness phase of the experiment. Positive and negative emotions affected driving change detection. P-emotion group perceived the changes faster than did $\mathrm{N}$ emotion group. Both emotions have decreased the accuracy of change detection. This suggests that both types of emotions are influential in change detection. In terms of reaction time, N-emotion group obviously required longer detection time than did P-emotion group. The effect of negative emotion is congruent with the emotion theory in the literature. According to this theory, negative emotions require more cognitive resources as a result of the longer time needed in the information processing. Therefore, the present study has demonstrated that positive emotions promote change detection and negative emotions interrupt the perceived changes in time. These results could infer that negative emotions decrease the speed of information processing with no effect on the accuracy of change detection.

Third, driving task effects were found in the final phase of experiment. There was no interaction effect between emotion and driving tasks. This finding is presumably due to the fact that positive and negative emotions have similar effects (accuracy of change detection) on driving behavior. The lack of interaction between emotion 
and driving tasks may be due to the small differences in performing different driving tasks. Therefore, under different driving tasks driver behavior does not substantially change for different emotions.

Nonetheless, driving tasks do have their own effects on change blindness. The results show that the signguided driving tasks caused an incremental effect in reaction time. The theory of cognition resources might be applied to explain these results. It is speculated that cognition resources are likely to be more occupied in the driving task under traffic signs than in those without traffic signs. Since the differences of the driving tasks under both conditions are too small to require more cognition processing in decision-making, there is not much decrease in the accuracy of change detection for both types of driving tasks.

The results show that the reaction time increment for RIGHT-task was much more than that for AHEAD-task. It is suspected that drivers are likely to spend more time in detecting dangerous stimuli when executing right turns. The results also found that the accuracy of change detection decreased much more dramatically in RIGHT- task than in AHEAD-task. It is postulated that right turns involve more difficulties to detect the safely threats from vehicles on the left side of the driver. The eye movement strategy may explain these results. The vision searching strategy is central, right, and left-orientation in this order (based on the rule of driver's seat on the left). Therefore, it is not difficult to understand that driver's attention would be devoted to the right side more than to left side, leading to a possible adverse effect on change detection.

\section{Conclusions}

This study has examined the effect of simultaneous emotions and driving tasks on change blindness. The experiment involved two types of emotions (happy and angry) combined with four driving tasks involving going ahead and turning right with and without guiding traffic signs. Based on this study, several comments are offered.

First, the negative emotion did affect the level of change blindness in terms of longer time of change detection. However, there was no significant difference between the P-emotion and $\mathrm{N}$-emotion groups in terms of accuracy of change detection. Besides, compared with the initial change blindness experiment, it was found that emotion had an effect on change blindness by decreasing the accuracy of change detection in the final experiment.

Second, emotion and driving tasks will likely influence driver change blindness. More specifically, negative emotions increase the reaction time in driving more than positive emotions do. Different driving tasks also had a significant effect on change blindness, where the tasks with signs took up more cognitive resources than did the tasks without signs. The turning-right tasks are more likely to suffer change blindness than do the going-ahead tasks. This finding would support the "No Right Turn on Red" practice at major signalized intersections, where right turns are allowed only when the respective travel directive receives a green signal indication.

Finally, this study has focused on the effect of emotion and four driving tasks (turning-right and going-ahead) with and without guiding traffic signs. Future research may address the effects of other driving tasks such as left-turn with permitted and protected traffic signals. Permitted left-turn movements are especially important since they involve a more complex environment that includes opposing vehicles and pedestrians competing for driver's attention.

\section{Acknowledgements}

The author would like to thank all collaborators for their advice and assistance. This research was financially supported by a research grant from Fuzhou University. The authors are grateful to all student assistants for their help in conducting the initial and final experiments of the study.

\section{References}

[1] Eby, D.W. and Kostyniuk, L.P. (2004) Crashes and driver distraction: A review of databases, crash scenarios, and distracted-driving scenarios. Transportation Research Institute, University of Michigan, Michigan, USA.

[2] Klauer, S.G., Dingus, T.A., Neale, V.L., Sudweeks, J.D. and Ramsey, D.J. (2006). The Impact of Driver Inattention on Near-Crash/Crash Risk: An Analysis Using the 100-Car Naturalistic Driving Study Data. National Highway Traffic Safety Administration, Washington DC.

[3] Van Elslande, P., Jaffard, M., Fouquet, K. and Vatonne, V. (2008) Variety of Attentional Failures in Traffic Accidents. European Conference on Cognitive Ergonomics, Funchal, 16-19 September 2008, 16-19.

[4] Simons, D.J. and Rensink, R.A. (2005) Change Blindness, Past, Present, and Future. Trends in Cognitive Sciences, 9, 
16-20. http://dx.doi.org/10.1016/j.tics.2004.11.006

[5] Rensink, R.A. (2002) Change Detection. Annual Review of Psychology, 53, 245-277. http://dx.doi.org/10.1146/annurev.psych.53.100901.135125

[6] O’Regan, J.K., Deubel, H., Clark, J.J. and Rensink, R.A. (2000) Picture Changes during Blinks: Looking without Seeing and Seeing without Looking. Visual Cognition, 7, 191-211. http://dx.doi.org/10.1080/135062800394766

[7] Caird, J.K., Edwards, C.J., Creaser, J.I. and Horrey, W.J. (2005) Older Driver Failures of Attention at Intersections: Using Change Blindness Methods to Assess Turn Decision Accuracy. Human Factors, 47, 235-249. http://dx.doi.org/10.1518/0018720054679542

[8] Koustanai, A., Elslande, P.V. and Bastien, C. (2012) Use of Change Blindness to Measure Different Abilities to Detect Relevant Changes in Natural Driving Scenes. Transportation Research Part F, 15, 233-242. http://dx.doi.org/10.1016/j.trf.2011.12.012

[9] Fredrickson, B.L. and Branign, C. (2005) Positive Emotions Broaden the Scope of Attention and Though-Action Repertoires. Cognition and Emotion, 19, 313-332. http://dx.doi.org/10.1080/02699930441000238

[10] Isen, A.M. (1990) The Influence of Positive and Negative Affect on Cognitive Organization: Some Implications for Development. In: Stein, N., Leventhal, B. and Trabasso, T., Eds., Psychological and Biological Approaches to Emotion, Hillsdale, Erlbaum, New Jersey, 75-94.

[11] Ashby, F.G., Isen, A.M. and Turken, A.U. (1999) A Neuropsychological Theory of Positive Affect and Its Influence on Cognition. Psychological Review, 106, 529-550. http://dx.doi.org/10.1037/0033-295X.106.3.529

[12] Liang, X.Y. and Yu, M. (2012) Influence of Emotion on Change Blindness. Clinical Psychology, 20, 32-34. (in Chinese).

[13] Galpin, A., Underwood, G. and Crundall, D. (2009) Change Blindness in Driving Scenes. Transportation Research Part F, 12, 179-185. http://dx.doi.org/10.1016/j.trf.2008.11.002

[14] Varakin, D.A., Levin, D.T. and Fidler, R. (2004) Unseen and Unaware: Implication of Recent Research on Failures of Visual Awareness for Human-Computer Interface Design. Human-Computer Interaction, 19, 389-422. http://dx.doi.org/10.1207/s15327051hci1904 9

[15] Levin, D.T. and Beck, M. (2004). Thinking about Seeing: Spanning the Difference between Metacognitive Failure and Success. In: Levin, D.T., Ed., Visual Metacognition: Thinking about Seeing, MIT Press, Cambridge, Massachusetts, 121-143.

[16] Most, S.B., Simons, D.J., Scholl, B.J., Jimenez, R., Clifford, E. and Chabris, C.F. (2001) How Not to Be Seen: The Contribution of Similarity and Selective Ignoring to Sustained Inattentional Blindness. Psychological Science, 12, 9-17. http://dx.doi.org/10.1111/1467-9280.00303 